\title{
Canonical Transformation of the Three-Band Hubbard Model and Hole Pairing
}

\author{
Michele Cini, Gianluca Stefanucci and Adalberto Balzarotti \\ INFM, Dipartimento di Fisica,Università di Roma Tor Vergata, Via \\ della Ricerca Scientifica 1- 00133 Roma, Italy
}

(May 27, 2018)

\begin{abstract}
We propose a canonical tranformation approach to the effective interaction $W_{\text {eff }}$ between two holes, based on the three-band Hubbard model but ready to include extra interactions as well. An effective two-body Hamiltonian can in principle be obtained including any kind of virtual intermediate states. We derive the closed-form analytic expression of the effective interaction including 4-body virtual states, describing the exchange of an electron-hole pair to all orders. The resulting integral equation, valid for the full plane, leads to a Cooper-like instability of the Fermi liquid. The two-hole bound states generalize those reported earlier in cluster calculations by exact diagonalisation methods.
\end{abstract}


The three-band Hubbard model Hamiltonian has often been used to characterize the electronic properties of high- $T_{C}$ superconductors [1] as observed in electron spectroscopy experiments. The model is:

$$
H=H_{0}+W
$$

where the independent hole hamiltonian reads, in the site representation

$$
H_{0}=\sum_{C u} \varepsilon_{d} n_{d}+\sum_{O} \varepsilon_{p} n_{p}+t \sum_{n . n .}\left[c_{p}^{+} c_{d}+h . c .\right]
$$

where n.n. stands for nearest neighbors. The on-site repulsion Hamiltonian will be denoted by

$$
W=\sum_{i} U_{i} n_{i+} n_{i-}
$$

where $U_{i}=U_{d}$ for a $\mathrm{Cu}$ site, $U_{i}=U_{p}$ for an Oxygen. We also considered first-neighbor O-O hopping and off-site interactions [2], but they were dropped when it was clear that they were not really important. The point symmetry Group of the $\mathrm{Cu}-\mathrm{O}$ plane is $C_{4 v}$, and its characters are listed in Table I. We wish to show that holes are paired in a Cooper-like fashion in the ground state of this popular model. Preliminarly, however, we must introduce the $\mathrm{W}=0$ pairs. Consider a determinantal 2-hole eigenstate of $H_{0}$ built from degenerate Bloch states. Can we superpose such wave functions to obtain simultaneous eigenstates of $H, H_{0}$ and $W$ ? For a general system, of course, we can't, but the symmetry of the problem enables us to achieve the result, and in addition to choose the eigenvalue $W=0$ of the on-site interaction. Indeed, omitting the band indices,let

$$
d[k]=\left\|k_{+},-k_{-}\right\|=c_{k,+}^{\dagger} c_{-k,-}^{\dagger} \mid v a c>
$$

be a two-hole determinantal state derived from the Bloch eigenfunctions of $H_{0}$. We introduce the determinants $R d[k]=d[R k]=d\left[k_{R}\right], R \in C_{4 v}$, and the projected states

$$
\Phi_{\eta}[k]=\frac{1}{\sqrt{8}} \sum_{R \in C_{4 v}} \chi^{(\eta)}(R) R d[k]
$$

where $\chi^{(\eta)}(R)$ is the character of the operation $R$ in the Irreducible Representation (Irrep) $\eta$. In the non-degenerate Irreps, the operations that produce opposite $k_{R}$ have the same character, and the corresponding projections lead to singlets. The explicit $\mathrm{W}=0$ singlet pair states are:

$$
\begin{array}{r}
\Phi_{1_{B_{2}}}\left[k, r_{1}, r_{2}\right]= \\
-\frac{\chi_{0}}{\sqrt{2}}\left\{\cos \left[k\left(r_{1}-r_{2}\right)\right] \phi\left(k, r_{1}\right) \phi\left(k, r_{2}\right)\right. \\
-\cos \left[k_{C_{4}}\left(r_{1}-r_{2}\right)\right] \phi\left(k_{C_{4}}, r_{1}\right) \phi\left(k_{C_{4}}, r_{2}\right) \\
-\cos \left[k_{\sigma}\left(r_{1}-r_{2}\right)\right] \phi\left(k_{\sigma}, r_{1}\right) \phi\left(k_{\sigma}, r_{2}\right) \\
\left.+\cos \left[k_{\sigma^{\prime}}\left(r_{1}-r_{2}\right)\right] \phi\left(k_{\sigma^{\prime}}, r_{1}\right) \phi\left(k_{\sigma^{\prime}}, r_{2}\right)\right\}
\end{array}
$$

and 


$$
\begin{aligned}
& \Phi_{1 A_{2}}\left[k, r_{1}, r_{2}\right]=\frac{\chi_{0}}{\sqrt{2}}\left\{\cos \left[k\left(r_{1}-r_{2}\right)\right] \phi\left(k, r_{1}\right) \phi\left(k, r_{2}\right)\right. \\
& +\cos \left[k_{C_{4}}\left(r_{1}-r_{2}\right)\right] \phi\left(k_{C_{4}}, r_{1}\right) \phi\left(k_{C_{4}}, r_{2}\right) \\
& -\cos \left[k_{\sigma}\left(r_{1}-r_{2}\right)\right] \phi\left(k_{\sigma}, r_{1}\right) \phi\left(k_{\sigma}, r_{2}\right) \\
& \left.-\cos \left[k_{\sigma^{\prime}}\left(r_{1}-r_{2}\right)\right] \phi\left(k_{\sigma^{\prime}}, r_{1}\right) \phi\left(k_{\sigma^{\prime}}, r_{2}\right)\right\} \text {, }
\end{aligned}
$$

where $\chi_{0}$ is a singlet spin function and $\phi$ is the periodic part of the Bloch function. Using the explicit Bloch states we can verify that both vanish for $r_{1}=r_{2}$. Therefore they have the $\mathrm{W}=0$ property. Turning now to the many-body problem, suppose the $\mathrm{Cu}-\mathrm{O}$ plane is in its ground state with chemical potential $\mu \equiv E_{F}$ and a couple of extra holes are added. We can consider the $\mathrm{W}=0$ pairs as bare quasiparticles for which no repulsion barrier needs to be overcome for pairing. Do the dressed quasiparticles look like Cooper pairs?

In Cooper theory [3], an effective interaction involving phonons is introduced via an approximate canonical transformation. Our problem is similar, except that the holes can exchange electron-hole pairs instead of phonons. Now the vacuum is the filled Fermi sphere and $\mathrm{W}$ connects the determinants $d[k]$ to the 4-body (3 hole-1 electron) determinants that carry no quasi-momentum; they are of the form

$$
|\alpha>=|\left\|\left(k^{\prime}+q+k_{2}\right)_{+}, \bar{k}_{2-},-q_{-},-k_{-}^{\prime}\right\|>
$$

where $\bar{k}_{2}$ is the electron state and pedices refer to the spin direction; those with opposite spin indices contribute similarly and yield a factor of 2 at the end. These are eigenstates of $H_{0}$ :

$$
H_{0}|\alpha\rangle=E_{\alpha}|\alpha\rangle
$$

The interaction matrix element reads:

$$
\begin{array}{r}
<\left\|\left(k^{\prime}+q+k_{2}\right)_{+}, \bar{k}_{2-},-q_{-},-k_{-}^{\prime}\right\||W| d[s]>= \\
\delta(q-s) U\left(q+k^{\prime}+k_{2},-k^{\prime}, s, k_{2}\right) \\
-\delta\left(k^{\prime}-s\right) U\left(q+k^{\prime}+k_{2},-q, s, k_{2}\right)
\end{array}
$$

where the $U$ matrix is computed on the Bloch basis. If we want to keep a close analogy with the Cooper theory, we temporarily consider $W$ as a small perturbation and look for an approximate canonical transformation such that the new Hamiltonian

$$
\tilde{H}=e^{-\Lambda} H e^{\Lambda}
$$

contains no first-order terms. Here, $\tilde{H}$ operates on the space of pairs, since the 3 holes- 1 electron intermediate states have been decoupled by the canonical transformation. This is accomplished if

$$
W+\left[H_{0}, \Lambda\right]=0
$$

because then, to second order,

$$
\tilde{H}=H_{0}+\frac{1}{2}[W, \Lambda] .
$$


Assuming that the denominators do not vanish (more about that later) we obtain

$$
\langle\alpha|\Lambda| s\rangle=\frac{\langle\alpha|W| s\rangle}{E_{s}-E_{\alpha}}
$$

We may write

$$
\tilde{H}=H_{0}+F+\tilde{W}_{e f f}
$$

where $F$ is diagonal in the pair space, like $H_{0}$, and corresponds to the unlinked self-energy diagrams, while the effective interaction operator is $\tilde{W}_{\text {eff }}$. Like in Cooper theory, F will be dropped. We obtain

$$
\begin{array}{r}
2\left\langle p\left|F+\tilde{W}_{\text {eff }}\right| s\right\rangle \\
=\sum_{\alpha} W_{p, \alpha} W_{\alpha, s}\left[\frac{1}{E_{p}-E_{\alpha}}+\frac{1}{E_{s}-E_{\alpha}}\right]
\end{array}
$$

Using the interaction matrix element (10), the product in (16) yields 4 terms; two are proportional to $\delta(p-s)$ and belong to $F$, while the cross terms yield identical contributions to the effective interaction.

After long algebra, we write the interaction between symmetry projected states (with $s$ and $p$ empty):

$$
\left\langle\Phi_{\alpha}[p]\left|\tilde{W}_{e f f}\right| \Phi_{\alpha}[s]\right\rangle=\sum_{R} \chi^{(\alpha)}(R)\left\langle d[p]\left|\tilde{W}_{e f f}\right| R d[s]\right\rangle
$$

where, explicitly,

$$
\begin{array}{r}
\left\langle d[p]\left|\tilde{W}_{e f f}\right| d[s]\right\rangle=2 \sum_{k}^{o c c} \Theta\left(\varepsilon(s+p+k)-E_{F}\right) \\
\times U(s+p+k,-p, s, k) U(p, k, s+p+k,-s) \\
\times\left[\frac{1}{\varepsilon(s+p+k)-\epsilon(k)-\epsilon(s)+\epsilon(p)}\right. \\
\left.+\frac{1}{\varepsilon(s+p+k)-\epsilon(k)-\epsilon(p)+\epsilon(s)}\right]
\end{array}
$$

In the continuum limit, the integral must be understood as a principal part, because the denominators vanish in a domain of zero measure within the integration domain. However, this complication is an artifact of the perturbation approach, as will soon become clear. The approximate canonical transformation is open to question because it assumes that $W$ be weak. Therefore, it is important to put the theory on a clearer and firmer basis, by removing the coupling to the $\alpha$ states to all orders.

The transformation (11, 12) corresponds to a second-order diagram for the two-hole amplitude, and if one of the hole lines is closed on itself one gets the corresponding self-energy. The expansion of $\Lambda$ can be continued systematically to produce the perturbation series. Including all the diagrams of a generalized RPA would lead to something like the well-known FLEX approximation [4] whose implications for the superconductivity in the three-band Hubbard model have been explored recently in a series of papers [5]. A related self-consistent 
and conserving T-matrix approximation has been proposed by Dahm and Tewordt [6] for the excitation spectra in the 2D Hubbard model; we mention incidentally that recently diagrammatic methods have been successfully applied to the photoelectron spectra of the Cuprates in other contexts too, like the spin-fermion model [7].

Realizing the key rôle of symmetry and $\mathrm{W}=0$ pairs in this problem enhances intuition besides simplifying the perturbation formalism considerably. Here, we wish to take advantage of the $\mathrm{W}=0$ pairs to propose a non-perturbative approach to pairing based on a different way of performing the canonical transformation. We write the ground state wave function with two added holes as a superposition of two-body states (roman indices) and 4-body ones:

$$
\left|\Psi_{0}>=\sum_{m} a_{m}\right| m>+\sum_{\alpha} b_{\alpha} \mid \alpha>.
$$

Schrödinger's equation then yields

$$
\begin{gathered}
\left(E_{m}-E_{0}\right) a_{m}+\sum_{m^{\prime}} a_{m^{\prime}} V_{m, m^{\prime}}+\sum_{\alpha} b_{\alpha} W_{m, \alpha}=0 \\
\left(E_{\alpha}-E_{0}\right) b_{\alpha}+\sum_{m^{\prime}} a_{m^{\prime}} W_{\alpha, m^{\prime}}=0
\end{gathered}
$$

where $E_{0}$ is the ground state energy. $V_{m^{\prime}, m}$ vanishes for $\mathrm{W}=0$ pairs in our model; however we keep it for generality, since it allows to introduce the effect of phonons [8,9] and any other indirect interaction [10 12 that we are not considering. Solving for $b_{\alpha}$ and substituting in (20) we exactly decouple the 4 -body states. The eigenvalue problem is now

$$
\left(E_{0}-E_{m}\right) a_{m}=\sum_{m^{\prime}} a_{m^{\prime}}\left\{V_{m, m^{\prime}}+\left\langle m\left|S\left[E_{0}\right]\right| m^{\prime}\right\rangle\right\}
$$

where

$$
\left\langle m\left|S\left[E_{0}\right]\right| m^{\prime}\right\rangle=\sum_{\alpha} \frac{<m|W| \alpha><\alpha|W| m^{\prime}>}{E_{0}-E_{\alpha}} .
$$

This is of the form of a Schrödinger equation with eigenvalue $E_{0}$ for pairs with an effective interaction $V+S$. Then we interpret $a_{m}$ as the wave function of the dressed pair, which is acted upon by an effective hamiltonian $\tilde{H}$. This is the canonical transformation we were looking for. However, the scattering operator $S$ is of the form $S=W_{\text {eff }}+F$, where $W_{\text {eff }}$ is the effective interaction between dressed holes, while $F$ is a forward scattering operator, diagonal in the pair indices $m, m^{\prime}$ which accounts for the self-energy corrections of the onebody propagators: it is evident that it just redefines the dispersion law $E_{m}$, and, essentially, renormalizes the chemical potential. Therefore $F$ must be dropped, as in Cooper theory [3] and above. Therefore the effective Schrödinger equation for the pair reads

$$
\left(H_{0}+V+W_{e f f}\right)\left|a>=E_{0}\right| a>
$$

and we are interested in the possibility that $E_{0}=2 E_{F}-|\Delta|$, with a positive binding energy $|\Delta|$ of the pair. The $V$ interaction just adds to $W_{e f f}$, and this feature allows to include in our model the effects of other pairing mechanisms, like off-site interactions, inter-planar 
coupling and phonons. Again, the product in the numerator of (23) yields 4 terms; two are proportional to $\delta(p-s)$ and belong to $\mathrm{F}$, while the cross terms yield identical contributions to $W_{\text {eff }}$. Hence we obtain the effective interaction between $\mathrm{W}=0$ pairs:

$$
\begin{array}{r}
\left\langle\Phi_{\eta}[p]\left|W_{e f f}\right| \Phi_{\eta}[s]\right\rangle=4 \sum_{R \in C_{4 v}} \chi^{(\eta)}(R) \sum_{k}^{o c c} \Theta\left(\varepsilon(R s+p+k)-E_{F}\right) \\
\times \frac{U(R s+p+k,-p, R s, k) U(p, k, R s+p+k,-R s)}{\varepsilon(R s+p+k)-\epsilon(k)+\epsilon(s)+\epsilon(p)-E_{0}}
\end{array}
$$

The sum is over occupied $k$ with empty $R s+p+k$. There are no vanishing denominators in the new expression, if $E_{0}$ is off the continuum. The $p$ and $s$ indices run over $1 / 8$ of the Brillouin Zone. We denote such a set of empty states $e / 8$, and cast the result in the form of a (Cooper-like) Schrödinger equation

$$
2 \varepsilon(k) a(k)+\sum_{k^{\prime}}^{e / 8} W_{e f f}\left(k, k^{\prime}\right) a\left(k^{\prime}\right)=E_{0} a(k)
$$

for a self-consistent calculation of $E_{0}$ (since $W_{\text {eff }}$ depends on the solution). Let $N_{C}$ be the number of cells in the crystal. The $U$ matrix elements scale as $N_{C}^{-1}$ and therefore $W_{\text {eff }}$ scales in the same way. For an infinite system, $N_{C} \rightarrow \infty$, this is a well defined integral equation. In principle, we can do better. By a canonical transformation one can obtain an effective Hamiltonian which describes the propagation of a pair of dressed holes, and includes all many-body effects. The exact many-body ground state with two added holes may be expanded in terms of excitations over the vacuum (the non-interacting Fermi sphere) by a configuration interaction:

$$
\left|\Psi_{0}>=\sum_{m} a_{m}\right| m>+\sum_{\alpha} b_{\alpha}\left|\alpha>+\sum_{\beta} c_{\beta}\right| \beta>+\ldots
$$

here $m$ runs over pair states, $\alpha$ over 4 -body states ( 2 holes and 1 e-h pair), $\beta$ over 6 -body ones ( 2 holes and 2 e-h pairs), and so on. To set up the Schrödinger equation, we consider the effects of the operators on the terms of $\left|\Psi_{0}\right\rangle$. We write:

$$
H_{0}\left|m>=E_{m}\right| m>, H_{0}\left|\alpha>=E_{\alpha}\right| \alpha>, \ldots
$$

and since $\mathrm{W}$ can create or destroy up to 2 e-h pairs,

$$
\begin{aligned}
W\left|m>=\sum_{m^{\prime}} V_{m^{\prime}, m}\right| m^{\prime}> & +\sum_{\alpha} \mid \alpha>W_{\alpha, m} \\
& +\sum_{\beta} \mid \beta>W_{\beta, m},
\end{aligned}
$$

and the like. The Schrödinger equation now gives an infinite system for the amplitudes of $2 \mathrm{n}-$ body states; however we can show [13] that these amplitudes can be successively decoupled, producing a renormalization of $\mathrm{W}$ matrix elements and energy eigenvalues $E_{\alpha}, E_{\beta}$ and so on. In principle, the method applies to all the higher order interactions, and we can recast our problem as if only 2 and 4-body states existed. If one calculates $W_{\text {eff }}$ neglecting 6-body and 
higher excitations, at least the structure of the solution is exact when expressed in terms of renormalized matrix elements.

The Hubbard model, with $\mathrm{V}=0$, leads to pairing, and the mechanism was first discovered in cluster studies [2]. Now we can better understand those results, since the above theory applies not only to the plane but also to clusters, provided that they are fully symmetric and allow $\mathrm{W}=0$ pairs. The symmetry of the cluster is essential, because only fully symmetric clusters allow such solutions. The planar lattice structure is also essential, because no $\mathrm{W}=0$ pairs occur in 3D or in a continuous model. We studied [2,14 16] the fully symmetric clusters with up to 21 atoms by exact diagonalisation. The main difference is that in the clusters the symmetries of $\mathrm{W}=0$ pairs were found [17, 18] to be ${ }^{1} B_{2}$ and ${ }^{1} A_{1}$. The reason for having ${ }^{1} A_{1}$ instead of ${ }^{1} A_{2}$ is a twofold size effect. On one hand, ${ }^{1} A_{1}$ pairs have the $\mathrm{W}=0$ property only in the small clusters, having the topology of a cross, and belonging to the $S_{4}$ Group, but do not generalize as such to the full plane, when the symmetry is lowered to $C_{4 v}$; on the other hand, those small clusters admit no solutions of ${ }^{1} A_{2}$ symmetry. To monitor pairing, initially we used [2] a definition of $\Delta$ taken from earlier cluster calculations [19,20] (where, however, only unsymmetric clusters were considered, and this mechanism could not operate):

$$
\Delta=E(N+2)+E(N)-2 E(N+1)
$$

where $E(N)$ is the ground state energy of the cluster with $\mathrm{N}$ holes, as obtained by exact diagonalisation. Pairing, that is, $\Delta<0$, was found when (and only when) the least bound holes formed a $W=0$ pair. In small clusters this conditions the occupation number, because the holes must partly occupy degenerate $(\mathrm{x}, \mathrm{y})$ orbitals, while in the full plane the $\mathrm{W}=0$ pairs (6, (7) exist at the Fermi level for any filling. The second-order approximation $\Delta^{(2)}$ was obtained by ground state energy diagrams (modified for degenerate ground states, when appropriate); the resulting expression [17] is clearly just the second-order approximation to the diagonal terms of (25). Then, we computed to second-order the two-hole amplitude for holes of opposite spins in the degenerate $(\mathrm{x}, \mathrm{y})$ orbitals. We demonstrated that this produced an effective interaction, which pushes down the singlet and up the triplet by $\left|\Delta^{(2)}\right|$. Good agreement between the second-order calculation and the numerical exact diagonalisation results supported the interpretation. Thus, we have shown that in the symmetric $\mathrm{Cu}-\mathrm{O}$ clusters genuine pairing takes place, due to an effective interaction which is attractive for singlets and repulsive for triplets. The cluster calculations [14-16] showed that $\mathrm{W}=0$ pairs are the "bare" quasiparticles that, when "dressed", become a bound state. That approach is inherently limited by the small size of solvable clusters, but allows a very explicit display of paired hole properties, that even show superconducting flux-quantization [17, 18].

The equations $(25,26$ ) allow to extend the study of pairs to the full plane. The integrand is very discontinuous because of Umklapp terms and of the limitations to occupied or empty states; moreover, its angular dependences are involved. The numerical solution is far from trivial, and the method that we developed will be presented elsewhere, with the numerical results. The main finding is the instability of the Fermi liquid in the model at hand. For both ${ }^{1} B_{2}$ and ${ }^{1} A_{2}$ we find pairing with a doping-dependent $\Delta$. The three-band Hubbard model might be too idealized to allow a detailed comparison with experiments; however we stress that contributions from phonons and other mechanisms can be included by a non zero $V$. The approach presented here is more general than the model we are using, and can be applied to realistic Hamiltonians. 
This work has been supported by the Istituto Nazionale di Fisica della Materia. We gratefully acknowledge A. Sagnotti, Università di Roma Tor Vergata, for useful and stimulating discussions. 


\section{REFERENCES}

[1] J.G. Bednorz and K.A. Muller, Z. Phys. B64 (1986) 189.

[2] M. Cini and A. Balzarotti, Il Nuovo Cimento D 18, 89 (1996).

[3] L.Cooper, Phys. Rev. 104,1189 (1956) .

[4] N.E.Bickers, D. J. Scalapino and S.R. White, Phys. Rev. Letters 62, 961 (1989); N.E.Bickers and S.R. White, Phys. Rev. B43, 8044 (1991).

[5] G. Esirgen and N. E. Bickers, Phys. Rev. Bbf 57, 5376 (1998) and references therein.

[6] T. Dahm and L. Tewordt, Phys. Rev. Letters 74, 793 (1995).

[7] J. Schmalian, D. Pines and B. Stojković, Phys. Rev. Letters 80, 3839 (1998).

[8] L. Pietronero and S. Strässer, Europhys. Lett18, 627 (1992).

[9] G. Iadonisi,M. Chiofalo, V. Cataudella and D. Ninno, Phys. Rev. B48, (1993) 12966 and references therein.

[10] J.R.Schrieffer, X.C.Wen and S.C.Zhang, Phys. Rev. Letters, 60, 944 (1988).

[11] P.W. Anderson, Science,235,(1987),1196; see also The Hubbard Model, Ed. D. Baeriswyl et al., Plenum Press, New York, 1995, page 217.

[12] O.P. Sushkov, Phys. Rev. B54, 9988 (1996) and reference therein.

[13] M. Cini, G. Stefanucci and A. Balzarotti, submitted for publication

[14] M. Cini and A. Balzarotti, J. Phys. Condens. Matter8 , L265 (1996).

[15] M. Cini and A. Balzarotti, Solid State Commun.101, 671 (1997).

[16] M. Cini, A. Balzarotti, J. Tinka Gammel and A. R. Bishop, Nuovo Cimento 19 D, (1997) 1329.

[17] M. Cini and A. Balzarotti, Phys. Rev. B56, 1, 14711 (1997).

[18] M. Cini, A. Balzarotti and G. Stefanucci, submitted for publication.

[19] J.E.Hirsch,S.Tang, E.Loh Jr, and D.J.Scalapino, Phys. Rev.Letters 60, 1668 (1988); Phys. Rev. B39, 243 (1989);

[20] C.A.Balseiro, A.G.Rojo, E.R.Gagliano, and B.Alascio, Phys.Rev.B 38, 9315 (1988). 


\section{TABLES}

\begin{tabular}{lllclcl}
\hline \hline $\mathrm{C}_{4 v}$ & $\mathrm{E}$ & $\mathrm{C}_{2}$ & $2 \mathrm{C}_{4}$ & $2 \sigma$ & $2 \sigma^{\prime}$ & \\
$\mathrm{A}_{1}$ & 1 & 1 & 1 & 1 & 1 & \\
$\mathrm{~A}_{2}$ & 1 & 1 & 1 & -1 & -1 & $R_{z}$ \\
$\mathrm{~B}_{1}$ & 1 & 1 & -1 & 1 & -1 & $x^{2}-y^{2}$ \\
$\mathrm{~B}_{2}$ & 1 & 1 & -1 & -1 & 1 & $x y$ \\
$\mathrm{E}$ & 2 & -2 & 0 & 0 & 0 & $(x, y)$ \\
\hline \hline
\end{tabular}

TABLE I. The Character Table of the $C_{4 v}$ Group 